\title{
Shaped beams: unlocking new geometry for efficient structures
}

\author{
Mohamed A. Ismail ${ }^{1}$ (1) - Paul L. Mayencourt ${ }^{1}$ - Caitlin T. Mueller ${ }^{1}$
}

Received: 22 March 2021 / Accepted: 1 August 2021 / Published online: 6 September 2021

(c) The Author(s), under exclusive licence to Springer Nature Switzerland AG 2021

\begin{abstract}
Society is building at an unprecedented rate: to house over 200,000 people moving to cities each day building stock will need to double by 2060. Importantly, the embodied carbon of construction due to material extraction, manufacturing, transportation, and demolition accounts for $11 \%$ of global carbon emissions, and this number is only expected to rise. With no end to construction in sight, it is essential that we develop better building practices. The research presented in this paper begins with the critical issue of embodied energy in horizontal structural systems. In high-rise buildings, between 60 and $80 \%$ of the mass and embodied energy of the structure can be found in the floors, suggesting a compelling starting point for materially efficient design. A reduction in a floor system's mass can lead to a similar reduction in the mass of vertical (columns, walls) and lateral systems. This paper focuses on the design of horizontal spanning elements, such as floor beams and slabs, and has two parts. The first part evaluates and compares historic methods of shaped beam design and classical methods of structural optimization. The second part presents a new flexible method of beam shape optimization. These design methods for structural efficiency allow us to build far more with far less, reducing the environmental and economic costs of construction while meeting the demands of a growing population.
\end{abstract}

Keywords Shape optimization $\cdot$ Beams $\cdot$ Structural analysis $\cdot$ Material efficiency $\cdot$ Historic methods

\section{Introduction}

\section{Motivation}

Society is building at an unprecedented rate: to house over 200,000 people moving to cities each day [1] building stock will need to double by 2060 , requiring the equivalent of a new Paris every week [2]. Importantly, the embodied carbon of construction due to material extraction, manufacturing, transportation, and demolition accounts for $11 \%$ of global carbon emissions. This number continues to rise as buildingrelated $\mathrm{CO}_{2}$ emissions grow by one percent per year [3]. With no end to construction in sight, it is essential that we develop better building practices. The research presented in this paper begins with the critical issue of embodied energy in horizontal structural systems.

In high-rise buildings, between 60 and $80 \%$ of the mass and embodied energy of the structure can be found in the

Mohamed A. Ismail

ismail@mit.edu

1 Department of Architecture, Massachusetts Institute of Technology, Boston, MA, USA floors [4], suggesting a compelling starting point for materially efficient design. A reduction in a floor system's self weight can lead to a similar reduction in the mass of vertical (columns, walls) and lateral systems. This paper has two parts focusing on the design of materially efficient horizontal spanning elements, such as floor beams and slabs. The first part evaluates and compares historic methods of shaped beam design and classical methods of structural optimization. The second part presents a new flexible method of beam shape optimization. These design methods for structural efficiency allow us to build far more with far less, reducing the environmental and economic costs of construction while meeting the demands of a growing population.

\section{Emerging opportunities in optimization and fabrication}

Structurally efficient design is not new, but it remains uncommon. Post-World War II, materials constrained construction costs while labor was plentiful and inexpensive. Consequently, architects and engineers around the world developed innovative materially efficient structures. This was especially true in developing countries where material 
costs encouraged efficient structures such as, but not limited to, long-spanning brick vaults by Eladio Dieste [5] and folded plate concrete roofs by Mahendra Raj [6]. These designers relied upon an understanding of material, construction, and mechanics coupled with an intuition for the flow of forces. As the cost of labor rose, however, interest in efficient structures gave way to standardized systems like flat slabs and prismatic beams [7]. Today, the looming climate crisis, a growing awareness of construction's embodied carbon emissions, and the growing selection of digital tools have revived interest in structural efficiency by incorporating structural analysis into the design and fabrication process.

In combination with modern structural optimization processes, contemporary digital fabrication methods, which use computer numerical control (CNC) devices like routers and 3D printers, can help materialize the complex results of structural optimization. No longer reliant upon affordable labor, digital fabrication enables the optimization of structural elements across a wide range of material systems. However, there is limited research on how these methods can translate to construction beyond one-off prototypes or boutique architecture. This paper's research is motivated by the relevance of shape optimization to the ubiquitous multistory urban construction occurring worldwide.

In contrast with design approaches inspired by nature, physical experiments, or complex numerical methods, this paper presents an approach for efficient flexural design routed in fundamental mechanics and established analytical expressions, amplified by computational geometry. The benefits of this are speed and ease of implementation, flexibility of geometric definition, and accuracy of physical behavior modeling. At the same time, because these proven methods can be directly linked to modern CAD environments, they benefit from the aforementioned opportunities in digital fabrication. This paper discusses the state of the art in existing analytical methods for beam shaping, and presents a new extension for shape optimization that combines historical methods of analysis with emerging tools in optimization and design. Finally, the paper presents several beam designs that highlight the flexibility of this method along with ongoing work that apply this paper's method to material systems like concrete slabs and timber beams.

\section{Existing methods of beam shape optimization}

Since Galileo Galilei's first analysis and shape optimization studies of a cantilever beam in 1638, many designers have been concerned with the full-utilization of structures by removing "waste" material. Also known as uniform strength beams, shaped beams are designed to minimize the amount of material necessary to resist a given loading envelope with given boundary conditions. As of this writing there is no comprehensive overview of historic shaped beam design methods. Consequently, this section discusses several existing methods and highlights their advantages and limitations in order to motivate further work on shaped beam design.

\section{History of mechanics and shape optimization}

In the 1638 text, Two New Sciences, Galileo's analytical attempt to understand the load carrying capacity of a cantilever beam led to his attempt at discovering the "solids of equal resistance" [8] and the first known attempt at designing a uniform strength beam.

As shown in Fig. 1, the result of Galileo's analytical method was the design of a cantilever beam with a parabolic reduction in depth along its length. Galileo's design of a uniform strength beam was ahead of its time; he also

Fig. 1 Galileo Galilea's design of a uniform strength beam, 1638

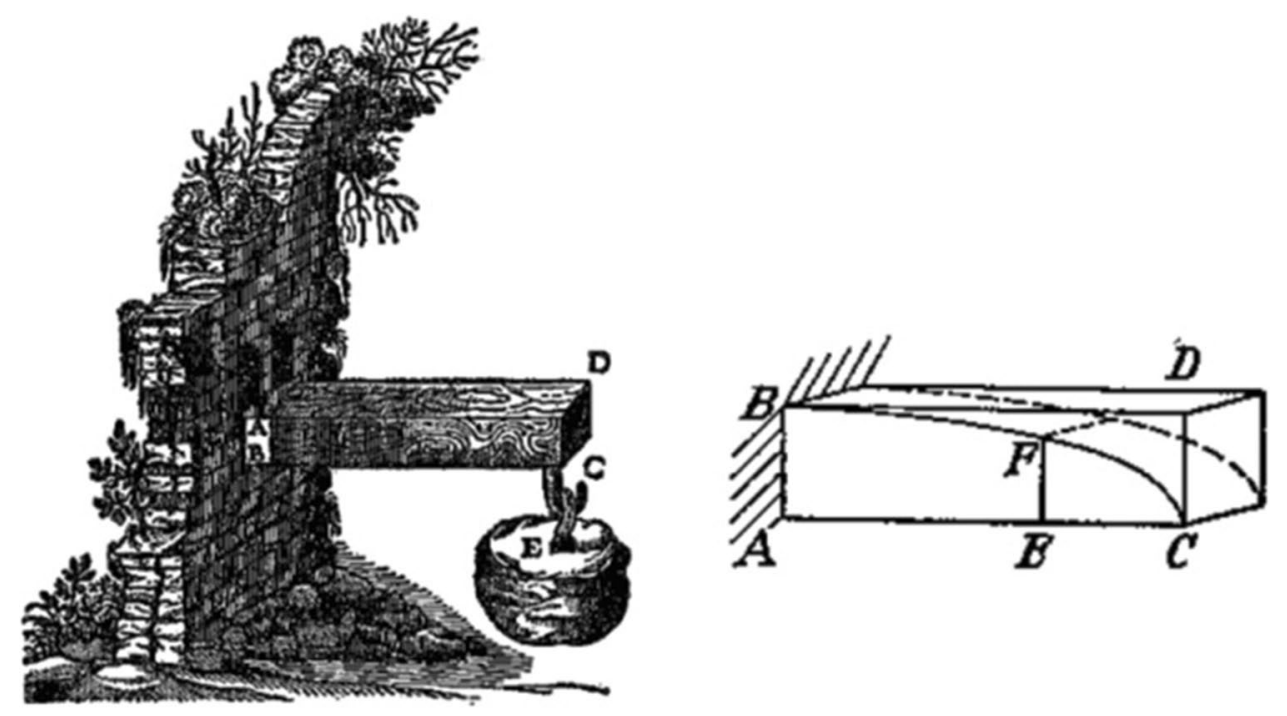


correctly observed that a beam with a hollow section could be stronger than a beam of similar mass but solid in section, preempting the use of hollow-core extruded sections in modern construction. Scientists continued to expand on Galileo's theorems over the following two centuries. Antoine Parent (1666-1716) was the first to determine the appropriate stress distribution within the section of a rectangular beam, leading to the well-known equation relating a beam's flexural strength-resistance to bending moments-to its geometry. Later on, Dmitrii Ivanovich Zhuravskii (1821-1891) found that beam shear was critical in the design of deep or composite beams, such as the built-up wood beams of railway bridges. While designing bridges for the St. Petersburg to Moscow railway in 1855, Zhuravskii derived the well-known equation relating a beam's geometry to its shear stress.

Galileo's problem of the uniform strength beam reappears in early to mid- twentieth century engineering handbooks which describe the design of a statically-determinate shaped beam, neglecting its self weight, as an elementary application of the flexural and shear strength formulas. In the seminal 1930 text, Strength of Materials, Timoshenko suggests that a beam be designed as a series of beams with different carrying capacities according to their strength requirements, leading to a segmented-rather than continuous-form [9]. Muttoni [10] explains this analytical approach in The Art of Structures by designing a number of uniform strength beams for a point load and comparing them over a range of slenderness ratios. These illustrations demonstrate a number of ways that shaped beams could be designed with varying efficiencies. Later advances in computation allow designers to revisit the problem of shaped structures with greater complexity and accuracy. Opatowski [11] designs a cantilever beam of uniform strength with a point load at its free-end, including the beam's self weight and requiring advanced integration. Li and Gross [12] discuss the design of uniform strength beams under a uniformly distributed load using Laplace transformations.

Around the 1960s, designers begin to apply the calculus of variation methods developed by Joseph-Louis Lagrange (1736-1813) to optimize the design of a beam subject to constraints. Barnett uses the calculus of variations to minimize the amount of steel needed in a statically-determinate steel I-beam [13]. Haftka, with reference to Barnett, outlines the procedure for designing a simply-supported beam subject to a deflection constraint [14]. Haug and Dupuis expand Barnett's work by including stress constraints [15, 16]. All of this work was limited to linearly elastic (linear relationship between stress and strain) and homogenous materials and stopped when the resulting designs became too complex for existing fabrication methods and construction practices.
Analytical solution: direct solve of uniform strength beams

Taking inspiration from Galileo's original approach, Barnett (1966) formalized a direct-solve (e.g. non-iterative) approach for beam shaping that calculates one variable dimension along a beam's length as an "insistence upon the proportional limit stress in the outer extreme fiber of the beam." Assuming linear elasticity and statically determinate conditions, this method results in a type of minimum-weight beam design that can be solved for without iteration if self weight is neglected [17]. This section discusses one such method based on Barnett's work for different variable dimensions and discusses the resulting volume reduction.

In this paper, simply-supported beams are designed with the material properties and boundary conditions detailed in Table 1. These material properties were chosen for a clear visualization of the geometric results and their differences. Additionally, the presented equations are specific to beams with solid rectangular sections but the overall method may be adapted to other section shapes with the appropriate equations for cross sectional properties. An example design process for a fixed-width, variable depth beam is summarized in Fig. 2.

In this paper's method, the goal is to find cross sectional geometries along the length beam that result in the least volume design without violating stress limits. A beam's volume $v$ is calculated by integrating the cross-sectional area along its length, as shown in Eq. (1):

$v=\int_{0}^{L} A(x) d x=\int_{0}^{L} b(x) h(x) d x$

where $A(x), b(x)$, and $h(x)$ are the area, width, and height of a section at location $x$ along the beam's length. It should be noted that one cross-section dimension, $b$ or $h$, must be held constant for a direct analytical solution to be found. Methods to find optimal expressions for $b(x)$ and $h(x)$ are given in the following sections.

Table 1 Material properties used in beam designs

\begin{tabular}{lrl}
\hline Property & Value & Unit \\
\hline Beam span & 3 & $\mathrm{~m}$ \\
Uniform external load & 20 & $\mathrm{kN} / \mathrm{m}$ \\
Material density & 5000 & $\mathrm{~kg} / \mathrm{m}^{3}$ \\
Yield strength (tension and compres- & 20 & $\mathrm{MPa}$ \\
$\quad$ sion) & 6 & $\mathrm{MPa}$ \\
Shear strength & 50 & $\mathrm{GPa}$ \\
Young's modulus & & \\
\hline
\end{tabular}


Fig. 2 Analytical design of a simply-supported beam with variable $h$ using similar method to [18]

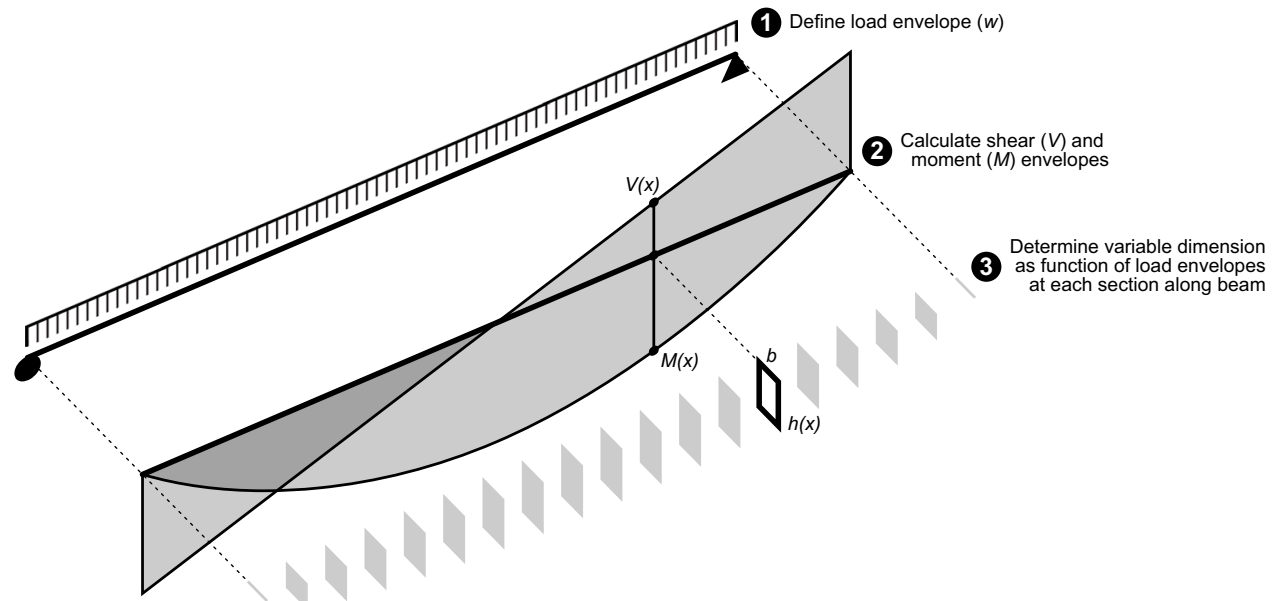

\section{Flexural design}

Flexural design for beam shaping starts with the basic mechanics of bending, which are given in the following paragraphs for completeness. The flexural stress at a given vertical location $y$ in a beam's section-at $x$ along its length-is expressed Eq. (2).

$\sigma(x)=\frac{M(x) y}{I(x)}$

where $x$ is the location along a beam's length, $\sigma(x)$ is the flexural stress at a distance $y$ from the beam section's neutral axis, $M(x)$ is the applied bending moment, and $I(x)$ is the moment of inertia of the beam's section.

The flexural stress distribution is shown in Fig. 3. Equating a material's yield stress $\sigma_{y}$ to the flexural stress at its most extreme fiber in Eq. (2) provides a method for shaping a beam according to the material's flexural strength.

The yield stress $\sigma_{y}$ is a property of the material, and the moment $M(x)$ is a function of the load envelope and boundary conditions. This leaves the moment of inertia $I(x)$ and the distance to the extreme fiber $y_{\max }$ as functions of the beam's variable dimensions. For a symmetrical rectangular beam section, the equation for its moment of inertia is given in (3):
$I=\frac{b h^{3}}{12}$

For convenience, it is possible to define a changing moment of inertia as Eq. (4):

$I(x)=\alpha A^{n}(x)$

where $\alpha$ and $n$ are constants related to the section's shape and fixed dimensions, and $A(x)$ is the variable area of the beam's cross-section along its length.

Using this formula, a number of derivations are possible for the design of shaped beams with fixed dimensions $b, h$, or the aspect ratio $\beta=h / b$. For instance, $n=1$ and $\alpha=h^{2} / 12$ corresponds to a rectangular section with constant depth $h$ and variable width $b(x), n=3$ and $\alpha=1 / 12 b^{2}$ corresponds to a rectangular section with constant width $b$ and variable depth $h(x)$, and $n=2$ and $\alpha=\beta / 12$ corresponds to a rectangular section with a constant aspect ratio $\beta$ and variable width and depth. Substituting these coefficients into Eqs. (3), (4) provides the solution for a variable dimension as a function of the other fixed dimensions and moment envelope. Consequently, the equation for the height of a constant width beam $(n=3)$ would be:
Fig. 3 Rectangular beam section undergoing a moment and the resultant flexural stress distribution in elevation

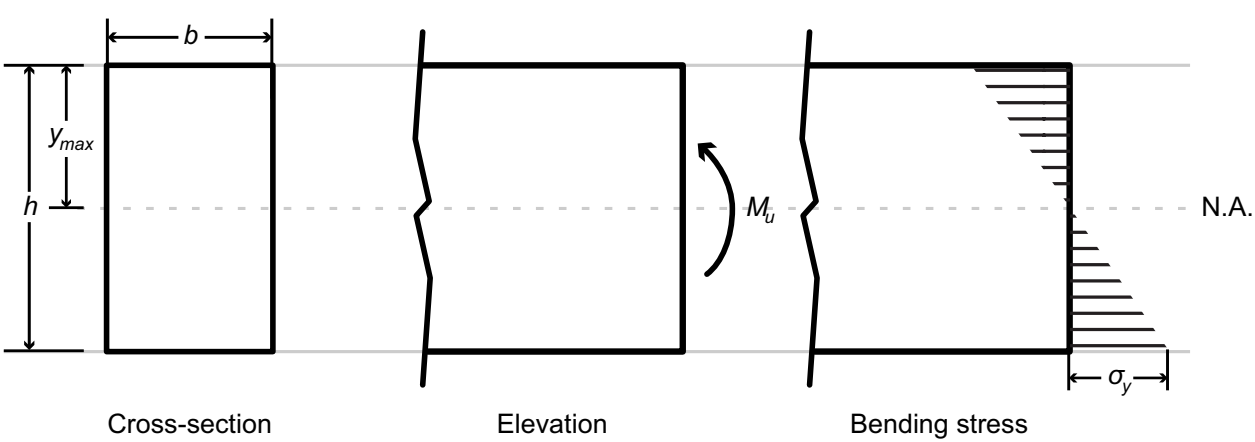


$h(x)=\sqrt{\frac{6 M(x)}{\sigma_{y} b}}$.

The equation for the width of a constant depth beam $(n=1)$ would be:

$b(x)=\frac{6 M(x)}{\sigma_{y} h^{2}}$.

These dimensions can also be expressed as functions of a constant aspect ratio $(n=2)$ :

$h(x)=\sqrt[3]{\frac{6 M(x) \beta}{\sigma_{y}}}$

$b(x)=\sqrt[3]{\frac{6 M(x)}{\sigma_{y} \beta^{2}}}$.

These equations for $h$ and $b$ define a type of structurally optimal beam with a rectangular cross section. Figure 4 illustrates the geometry of the beams determined using Eqs. (5), (8) and the design of a typical prismatic beam designed for the maximum flexural stress. Importantly, the shaped beams have a depth or width of zero where the bending moment is zero, resulting in the probable shear failure in built structures. It is therefore essential to consider the shear capacity of the beam, as described in the following section.

\section{Shear design}

While flexural stresses due to bending often control the design of long and slender beams, it is also important to account for shear stress (see Fig. 5).

The following derivation is theoretically valid for a shaped beam with small changes in section, but there is literature that offers a more realistic method for the shear stress analysis of shaped beams [19-21] as shown in Fig. 5. Thus, the relevant formula relating a material's maximum shear stress $\tau_{\max }$ to a section's geometry is:
Fig. 4 Result of analytically shaping a $3 \mathrm{~m}$ beam according to flexural stress

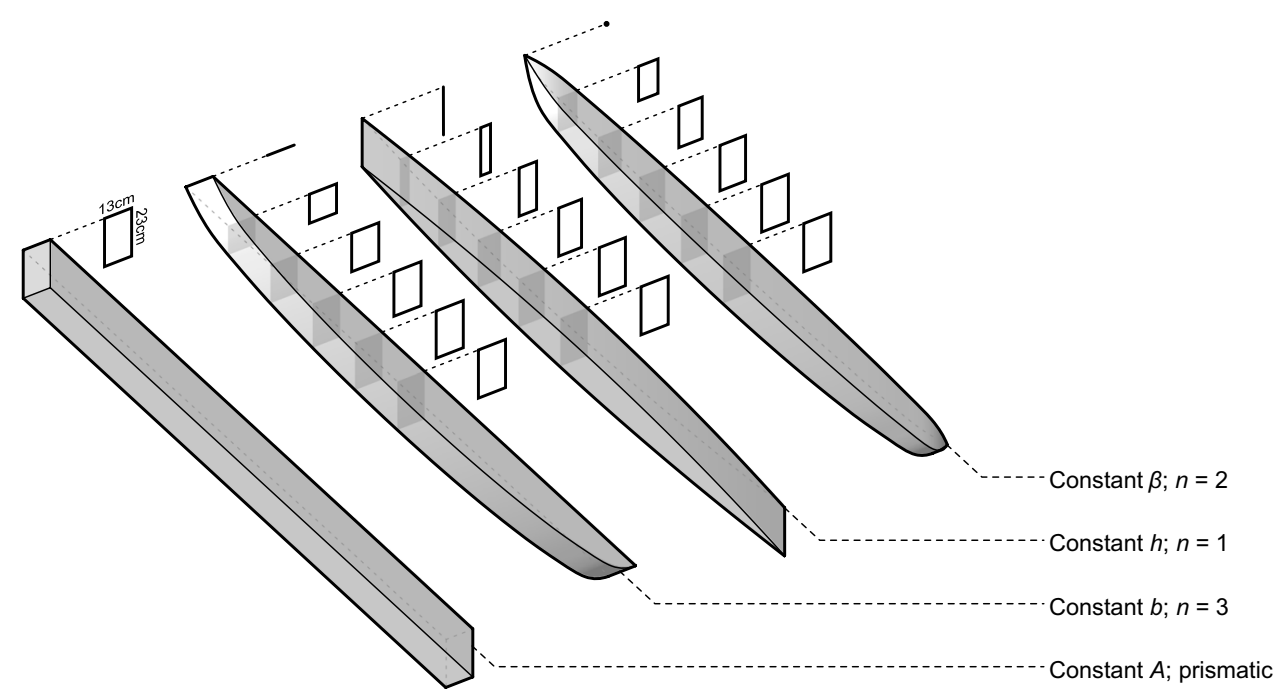

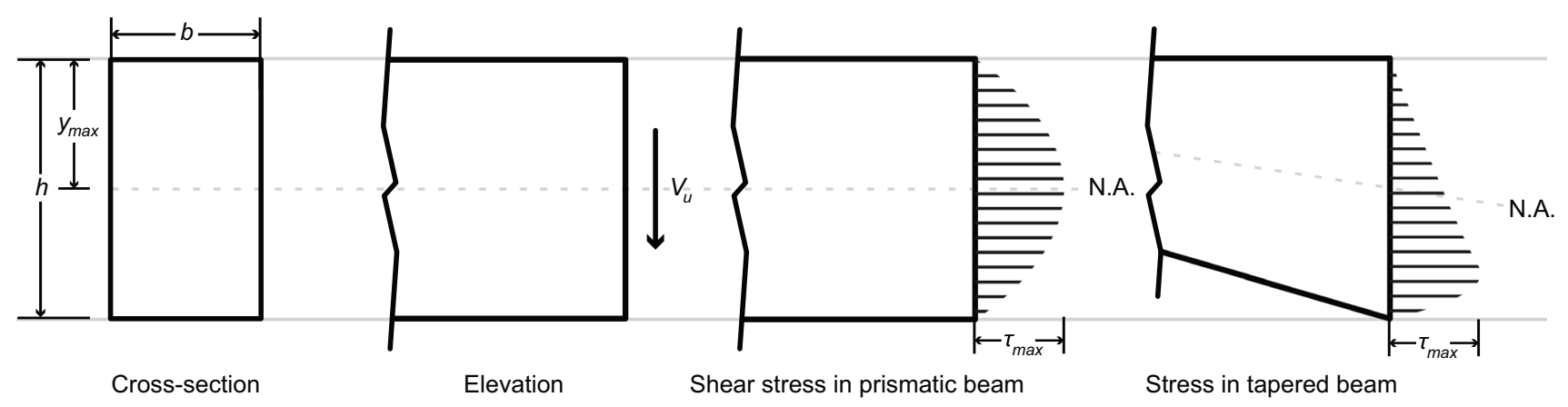

Fig. 5 Rectangular beam section undergoing a shear force and the resultant shear stress distribution for a prismatic and tapered (shaped) beam 
$\tau_{\max }=\frac{V(x) Q_{\max }(x)}{I(x) b}$

where $\tau_{\max }$ is the maximum shear stress at location $x$ along the beam's length, $V(x)$ is the applied shear force, $Q_{\max }(x)$ is the first moment of the beam's section, and $b$ is the beam's width.

In a vertically loaded beam, the assumed maximum shear force occurs at the neutral axis (see Fig. 5), where $y=0$. Therefore, the value of the first moment of area $Q_{\max }$ for a symmetrical rectangular section is:

$Q_{\max }=\int_{0}^{h / 2} y d A=\frac{b h^{2}}{8}$.

Along with Eq. (4), the above equation can be substituted into Eq. (9) to relate the dimensions of a rectangular section to the material's maximum shear stress and the shear force envelope. This can then be re-arranged to calculate a beam's depth or width as a function of the other dimension, or the aspect ratio $\beta$, as in Eqs. (11), (12):

$h(x)=\frac{3}{2} \frac{V(x)}{\tau_{\max } b}=\sqrt{\frac{3}{2} \frac{V(x) \beta}{\tau_{\max }}}$

$b(x)=\frac{3}{2} \frac{V(x)}{\tau_{\max } h}=\sqrt{\frac{3}{2} \frac{V(x)}{\tau_{\max } \beta} .}$

These equations define a beam geometry that is optimal for shear resistance only (see Fig. 6), resulting in beams with little to no flexural resistance. In practice, both flexure and shear stresses must be considered, as discussed in the following section.

\section{Combining flexure and shear for beam shaping}

In order to account for both flexure and shear, a controlledwidth beam can be designed using the controlling dimension for both forms of internal stress. For example, the full Eq. (13) for a constant width beam's depth would be:

$h(x)=\max \left(\sqrt{\frac{6 M(x)}{\sigma_{y} b}}, \frac{3}{2} \frac{V(x)}{\tau_{\max } b}\right)$.

Similar equations can be derived for a controlled-depth or constant-aspect ratio beam. Each of these formulas result in a different type of uniform-strength beam with differing degrees of material efficiency as illustrated in Fig. 7.

The graphs in Fig. 8 compare each type of shaped beam to a traditional prismatic (e.g. extruded, constant cross section) element for a range of slenderness ratios, $L / h$, where $L$ is the span of the beam and $h$ is its maximum depth. The beams are designed for simply supported boundary conditions undergoing a uniform line load $w$ so the shear and moment envelopes are calculated using Eqs. (14), (15):

$V(x)=w\left(\frac{L}{2}-x\right)$

$M(x)=\frac{w x}{2}(L-x)$.

For a dimensionless comparison, the volumes of the resulting optimal beams are normalized to a quantity $v_{\text {norm }}^{*}$ to account for the span $L$, yield stress $\sigma_{y}$, and applied load $w$ using Eq. (16).

$v_{\text {norm }}^{*}=\frac{v^{*}}{w L^{2} / \sigma_{y}}$
Fig. 6 Result of analytically shaping a $3 \mathrm{~m}$ beam according to shear stress

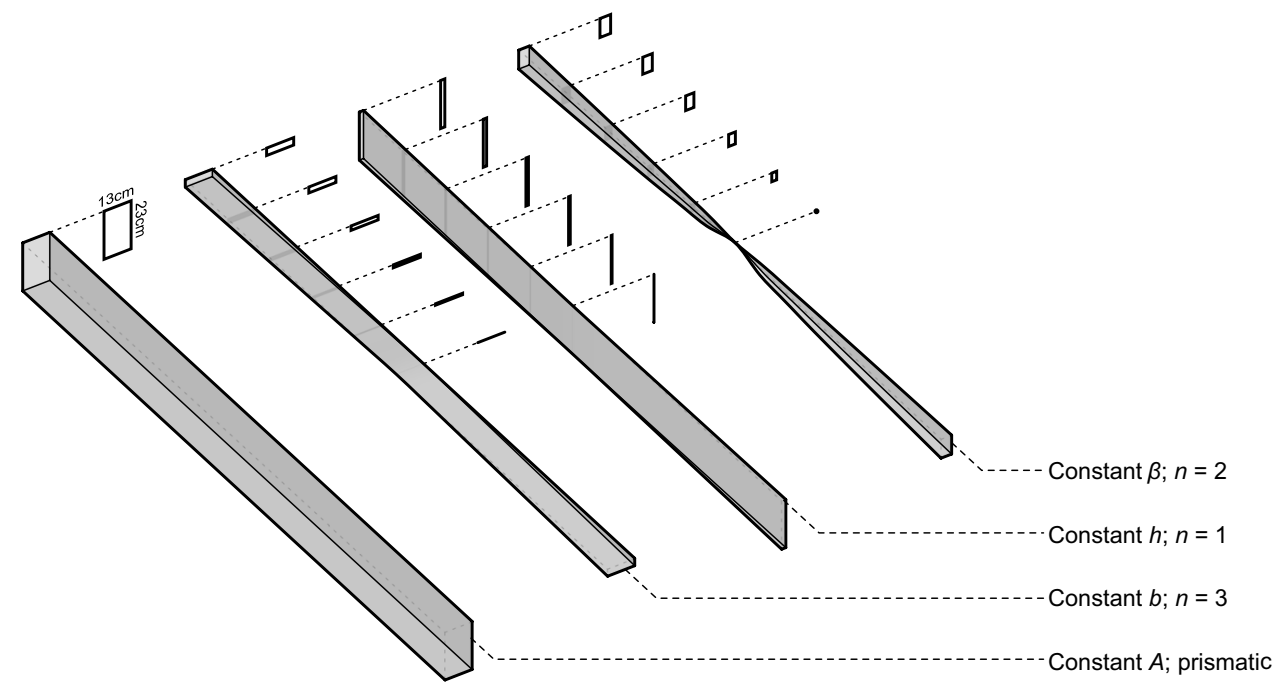


Fig. 7 Result of analytically shaping a $3 \mathrm{~m}$ beam according to flexure and shear stress

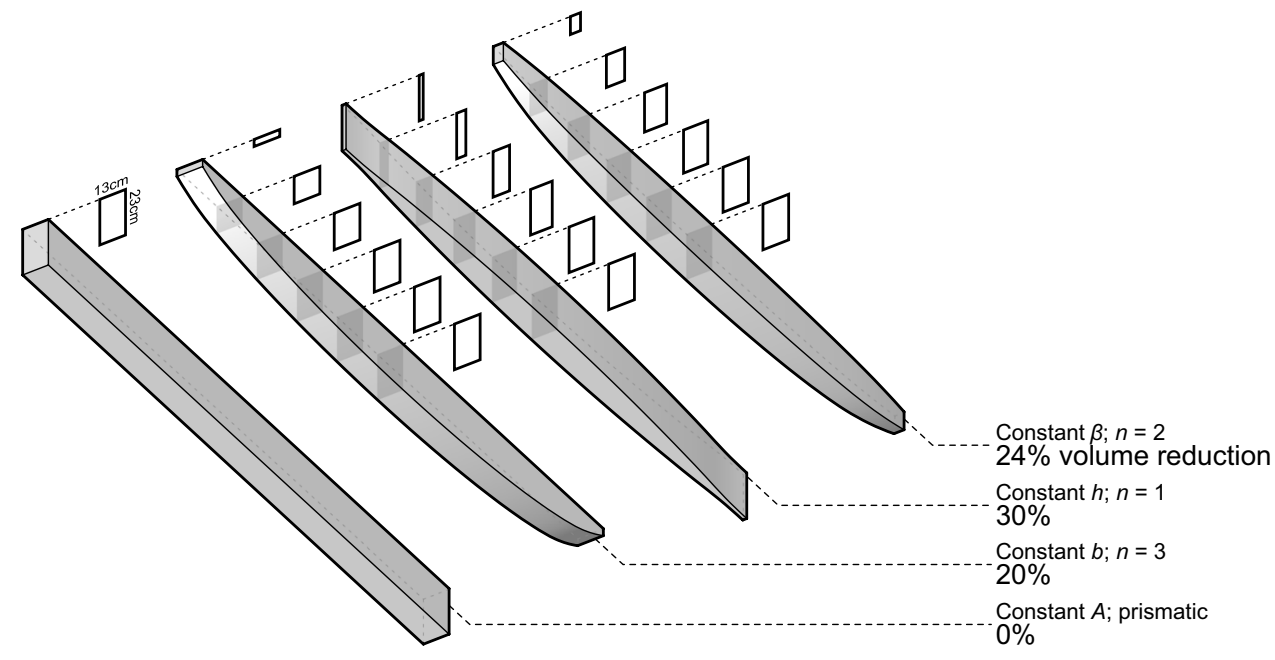

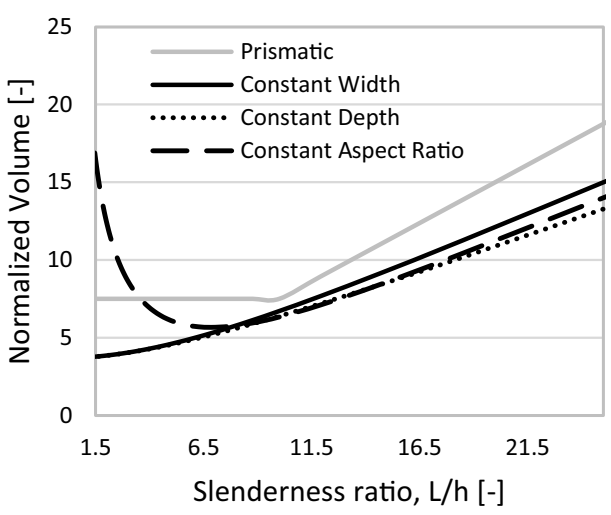

a) $\tau_{\max }=0.15 f_{y}$, similar to wood

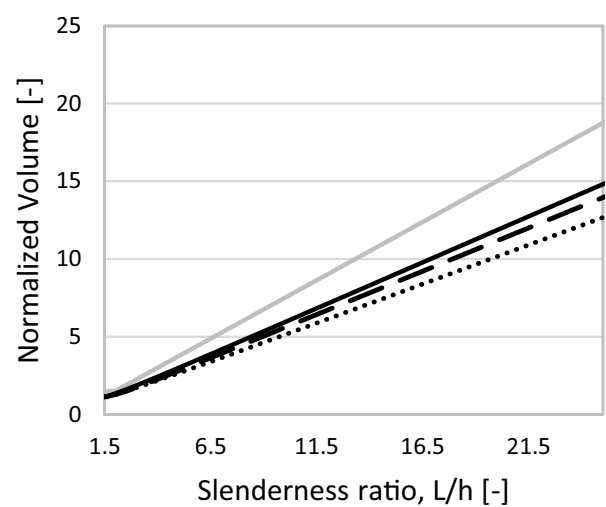

b) $\tau_{\text {max }}=0.50 f_{y}$, similar to steel
Fig. 8 Volume of material in relation to the slenderness ratio $L / h$ with a shear strength that is a) $15 \%$ of the flexural strength and b) $50 \%$ of flexural strength (referencing Muttoni [10]) where $v^{*}$ is volume of the optimal design, computed using Eq. (1).

The graphs also show the impact of a material's shear strength on the volume of a shaped beam, especially for deep beams (low slenderness ratio) where shear stress becomes critical. These plots show the volume of a shaped beam with a shear strength that is $15 \%$ (similar to wood) and $50 \%$ of its flexural strength (similar to steel). There is a point of inflection where each type of shaped beam shifts from shear-controlled to flexure-controlled designs. This explains the increased volume for low slenderness ratios, especially when the material's shear strength is considerably lower than the flexural strength (as in Fig. 8a). Many designers believe the most efficient beams have a depth that is proportional to the applied moment, but these results show that this is not always the case; more efficient designs can be achieved through other means, such as shaping the width. For slenderness ratios between 5 and 25 and materials with larger shear strengths (such as in Fig. 8b), the volume reduction is approximately $20 \%$ for the constant width beam, $25 \%$ for the constant aspect ratio beam, and $30 \%$ for the constant depth beam.

This historical direct-solve method is a useful tool that relies on simple operations, but it cannot be used for more complex design and engineering scenarios. For example, varying both the width and the depth of the beam independently is not possible with this method, nor is it possible to account for constraints such as maximum deflection. In response to some of these limitations, the following section presents more recently developed methods that address more complex design problems.

\section{Alternative methods}

\section{Analytical optimization of shaped beams}

The previous section discusses a method that relates the applied load to a variable dimension. Alternatively, variational calculus can be used to find the maximum or minimum value of an objective function subject to structural 
constraints. In an unconstrained problem-with a twicedifferentiable objective function-critical values are points where the derivative of the function is equal to zero. These are stationary points that mark the local maxima or minima of the function. As discussed by Barnett [13] and Haftka [14], Lagrange multipliers can help transform a constrained problem into an unconstrained one. This allows designers to include constraints, such as a fixed volume of material or a maximum deflection, in the design of a shaped beam. Haftka's method for the design of a constrained beam subject to an equality constraint on its maximum deflection begins with the following Eqs. (17), (18):

$$
\underset{A(x)}{\operatorname{Minimize}} \quad v=\int_{0}^{L} A(x) d x
$$

Subject to $\Delta(\xi)=\Delta_{0}$

where $\Delta(\xi)$ is the deflection at $x=\xi$, and $\Delta_{0}$ is the desired maximum deflection.

Summarizing Haftka's derivation and using the method of virtual work, the deflection at $x=\xi$ is found using the Eq. (19):

$\Delta(\xi)=\int_{0}^{\xi} \frac{M(x) m(x)}{E I(x)} d x$

where $m(x)$ is the "virtual" moment of a point load applied at the location $x=\xi$, and $E$ is the Young's modulus of the material.

The moment of inertia $I(x)$ is again rewritten using Eq. (5). Setting $n=1$ for convenience, Haftka derives the optimum area distribution $A^{*}(x)$ subject to a maximum deflection of $\Delta_{0}$. This is carried out using Lagrange multipliers and results in the following Eq. (20).

$A^{*}(x)=\frac{1}{\alpha E \Delta_{0}}\left[\int_{0}^{l}(M(\eta) m(\eta))^{\frac{1}{2}} d \eta\right](M(x) m(x))^{\frac{1}{2}}$

Similar to the method described in the previous section, this function can be used to define a beam geometry by dividing $A^{*}(x)$ by a fixed width or length, resulting in the variable dimension's functional definition. This equation is only useful for the design of a cantilevered beam subject to a deflection equality constraint at its free end, highlighting the limits of this type of analytical shaped beam optimization.

\section{Numerical shape optimization}

In contrast to the analytical methods described above, numerical shape optimization provides the freedom to design structures with innumerable geometries, load cases, and boundary conditions [22]. A number of researchers have developed iterative methods that couple finite element analysis (FEA), a common numerical method of structural analysis, with numerical optimization algorithms for the design of efficient 2D and 3D structures [23, 24]. There are also methods that pair FEA with iterative optimization algorithms to design efficient concrete beams [25, 26].

While these methods work well with CAD software and digital fabrication technologies, numerical shape optimization has a number of challenges that include: the definition of appropriate mesh densities, sensitivity to changing boundary conditions, and lengthy computation times when FEA is used to assess the performance of variable-shaped structures.

\section{Limitations and opportunity for shape optimization}

Research in the design of uniform-strength beams results in interesting closed-form solutions that provide rapid solutions to structural efficiency problems. Yet, they are often difficult to define, do not always exist for complex conditions or geometries, and are limited to one problem definition. For instance, the analytical solution to minimize the volume of a circular-section cantilever beam with a maximum deflection constraint has one solution for a point load at its end that cannot be used for any other loading condition or section geometry [14].

The analytical optimization of a shaped beam may lead to an exact solution but it is often time consuming, difficult, and infeasible for complex problems that more closely relate to real applications. Moreover, an analytical approach to optimization is impossible when there is no formula linking the open variables to the final objectives and constraints. This occurs when CAD software like Rhino is used for geometric designs that are detached from an objective formula like material volume. It is, in fact, rare to find a practical structural design problem that can be solved using a purely analytical optimization.

As demonstrated in this paper, there are a number of methods to design a shaped beam and the choice varies according to the designer's objective, fabrication limits, material system, and more. In response to the limitations outlined here, this paper presents a new method that pairs immediate analytical formulations with numerical shape optimization to rapidly design shape optimized beams subject to structural and fabrication constraints.

\section{A new approach to beam shaping}

This section discusses the design of a beam with a changing rectangular section, referencing the analytical solutions discussed by Muttoni and Timoshenko and described in detail in the previous section. This method focuses on the design of a shaped beam for volume minimization and assumes the material is homogenous and linear elastic with the properties 
described in Table 1, carrying a continuous distributed load with simply supported boundary conditions.

\section{Numerical optimization}

As opposed to analytical methods, numerical methods of optimization use algorithms that iteratively approach an optimum through approximation. These algorithms will often terminate after a set number of steps or after the iterations converge near a solution within an acceptable tolerance. A growing number of tools allow designers to enhance their design workflow in CAD environments such as Rhino with numerical optimization, including toolkits like Galapagos, Goat [27], and Design Space Exploration, abbreviated DSE [28].

These tools can help designers design shaped structures using constrained numerical optimization. This section describes one such method: the shape optimization of a minimum-weight shaped beam subject to shear and moment constraints. The geometric workflow is illustrated in Fig. 9 below.

Using Rhino's geometry library, the volume of the beam is calculated as the objective for optimization. An array of sections is extracted and analyzed against the moment and shear envelope. This analysis occurs at numerous points to check that the beam meets the changing structural demands along the beam's length; these checks are the constraints for optimization. This analysis uses the equations discussed in "Constraints" section, making this a hybrid method of analytical analysis and numerical optimization. To reduce the computational demand, this method analyzes just half of the beam due to symmetry. All of this is done using DSE's constrained optimization tool, Radical, and the COBYLA algorithm [29].

Unlike the analytical method, the algorithm is simultaneously shaping the beam's width and depth along its length in response to the moment and shear demands. This results in unique designs that would not be possible without NURBSbased geometry representation, and can result in more materially efficient shaped beams.

\section{Variables}

In the design of a shaped beam with a rectangular section, the variables may include parameters such as the beam's height, width, or aspect ratio.

The variable parameters depend on the geometric topology of the beam, so designers can use methods similar to those described in this paper to design shaped beams with a variety of geometries as long as the formulas are adapted to their geometric properties (see Fig. 10). In this paper, the design of a shaped beam is defined with a series of rectangular sections (Fig. 10) along the length of the beam with variable widths and heights.

First, the span, boundary conditions, material properties, and load envelope are defined. Then, rectangles with variable dimensions (see Fig. 11) are arrayed along half the length of the beam; their dimensions are the variables for optimization. Symmetry is assumed about the midpoint of the span, reducing the necessary number of variables for optimization. For the sake of comparison, these variables are bounded by the dimensions of an equivalent prismatic beam, but better results are possible when those bounds are relaxed. The beam's geometry is then constructed by lofting the section curves [30] to create
Fig. 9 Workflow for the digital design and optimization of a shaped beam

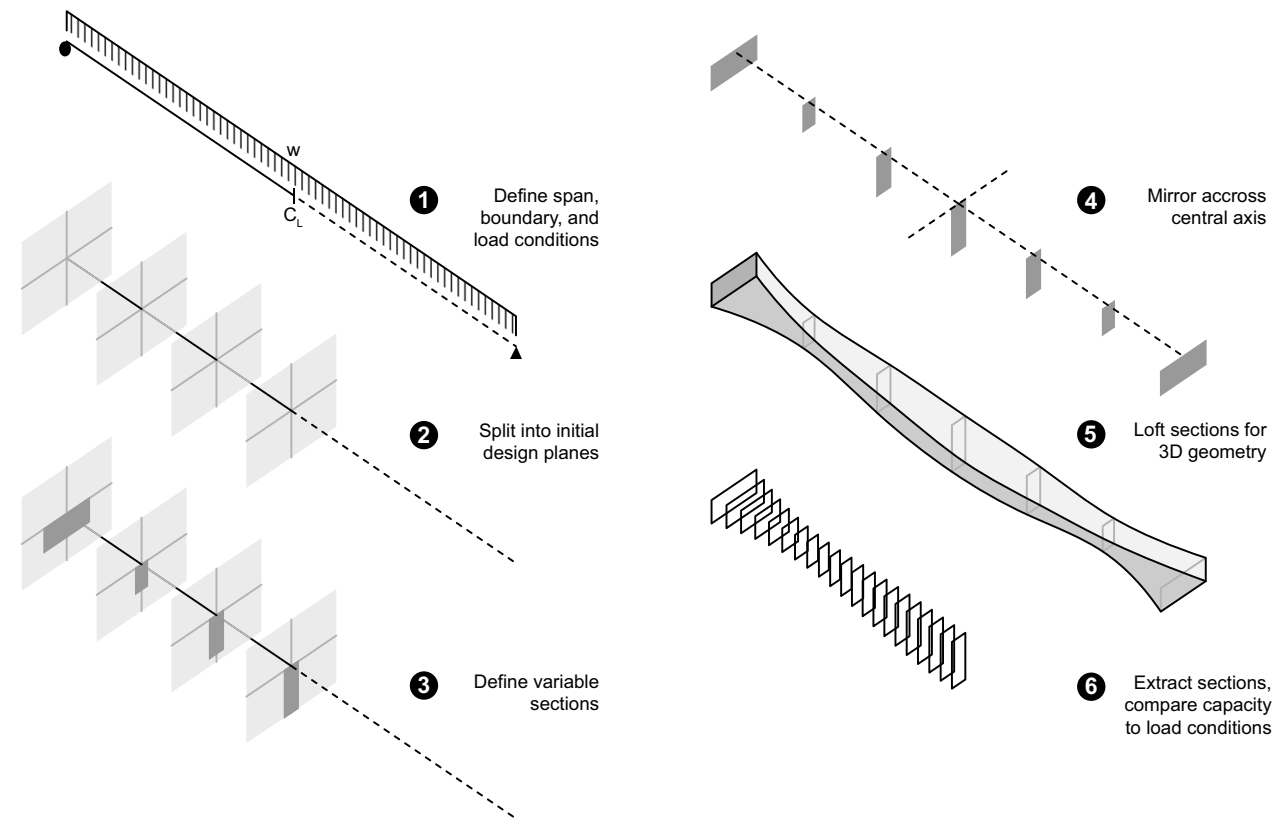




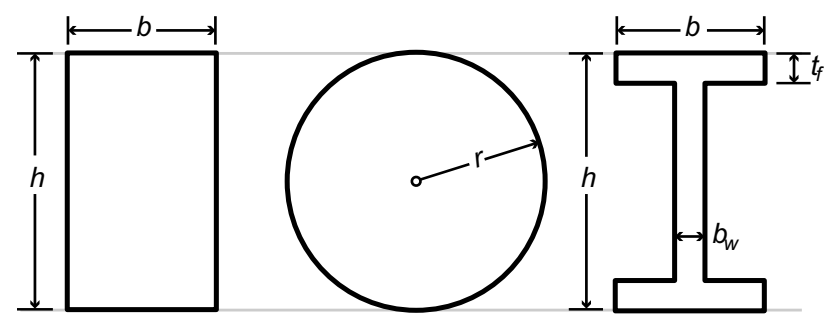

Fig. 10 Variable dimensions for (from right to left) a rectangular, circular, and wide-flange section beam

a solid geometry that can be analyzed as described in "Constraints" section. Smooth geometry from the lofting operation helps avoid shear concentrations from sudden changes in section [9]. The sections are lofted together using Rhino's geometry library.

\section{Objectives}

The objective can involve volume minimization [11], strength maximization [31], stiffness maximization [32], span maximization and more. An example design problem may be to maximize the span for a given material volume, setting the beam's volume as a constraint and the span as the objective. The objective can be contextual, like the cost of materials in developing economies or the cost of labor and fabrication complexity in more developed economies [33]. For the purposes of this paper, the objective is the volume of a simplysupported beam. Thus, the optimization problem is defined using Eq. (21).

$\underset{\boldsymbol{a}}{\operatorname{Minimize}} v(\boldsymbol{a})$

$\boldsymbol{a}=\left[\begin{array}{c}b_{1} \\ h_{1} \\ \vdots \\ b_{N} \\ h_{N}\end{array}\right]$

\section{Subject to $g(\boldsymbol{a}) \leq 0$}

where $v$ is the beam's volume as a function of the design variable vector $\boldsymbol{a}, N$ is the number of sections defining the beam's geometry, and $\boldsymbol{g}(\boldsymbol{a})$ is the vector of constraints that must be met during optimization. It is important to note that while there is no direct analytical expression for volume $v$ this method uses Rhino's geometry library to automatically determine the volume of a lofted beam geometry.

In order to verify that a shaped beam meets the desired structural (or non-structural) conditions, the minimization function is subject to a number of inequality constraints,

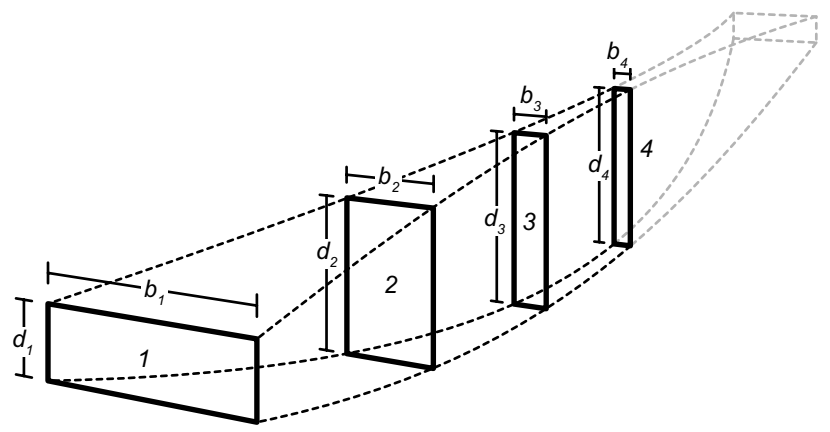

\begin{tabular}{|lll|}
\hline Variable & Definition & Bounds \\
\hline $\boldsymbol{b}_{\boldsymbol{i}}$ & Width of section $i$ & $0.005 \mathrm{~m} \leq b_{i} \leq 0.100 \mathrm{~m}$ \\
\hline $\boldsymbol{d}$ & Depth of section $i$ & $0.005 \mathrm{~m} \leq d_{i} \leq 0.260 \mathrm{~m}$ \\
\hline
\end{tabular}

Fig. 11 Example variable setup and their bounds for beam shape optimization, "Results" section marks the midpoint of the beam

$\boldsymbol{g}(\boldsymbol{a})$. Examples of such constraints are discussed in the following section.

\section{Constraints}

The design of a shaped beam involves constraints like maximum deflection or stress [16], or dimensional constraints like the maximum depth permitted by the architecture. Constraints can account for particular material properties, behaviors, and failure modes relevant to a wide variety of construction materials (e.g. reinforced concrete, glue-laminated timber, etc.) Constraints can also be non-structural, such as fabrication complexity or aesthetic considerations. An example of a fabrication constraint may be the curvature of a beam's surfaces, allowing a designer to design a beam with singly-curved surfaces for fabrication means like robotic wire-cut foam or bent plywood.

While it is clear that constraints can extend well beyond the structural considerations in the design of a shaped beam, this paper focuses on the structural constraints of shear and flexural resistance. As discussed earlier, the moment and shear capacity of a beam's section can be analytically determined using its dimensions and material properties. Rearranging Eqs. (1), (2) would give us, respectively, the Eqs. (22), (23) for a rectangular beam section's shear $V_{n}$ and moment $M_{n}$ capacity:

$V_{n}=\frac{2}{3} * \tau_{y} b h$

$M_{n}=\frac{1}{6} * \sigma_{y} b h^{2}$

where $\tau_{y}$ and $\sigma_{y}$ are, respectively, the yield shear and bending stress of the beam's material. 
In order to verify that a shaped beam meets the full shear and moment envelope, the optimization problem is subject to shear and moment constraint checks at multiple sections along the beam's length. These constraints are checked at each iteration of the optimization and inform the optimization as it approaches a minimum volume. Importantly, this optimization problem may involve many constraints depending on number of sections chosen for analysis and the number of constraints considered at each section. Depending on the algorithm, the shear and moment constraints at section $i$ may be defined as in Eqs. (24), (25):

$M_{n, i}-M_{u, i} \leq 0$

$V_{n, i}-V_{u, i} \leq 0$

where $M_{n, i}$ and $V_{n, i}$ are the moment and shear capacities, and $M_{u, i}$ and $V_{u, i}$ are the factored external moment and shear at beam section $i$.

In comparison to equivalent strength prismatic beams that have a continuous stiffness along their length, serviceability may play a significant role in the design of shaped beams which may lose stiffness due to reduced section sizes. In this paper, serviceability is defined as the maximum immediate deflection of a beam under external loading. According to the Euler-Bernoulli beam theory, the rotation $\theta(x)$ and vertical deflection $\Delta(x)$ of an elastic beam at location $x$ along its length are functions of the applied moment $M(x)$, as in Eqs. (26), (27).

$\theta(x)=\int \frac{M(x)}{E I(x)} d x+C_{1}$

$\Delta(x)=\int \theta(x) d x+C_{2}$

where $E$ is the beam material's Young's Modulus. $C_{1}$ and $C_{2}$ are the constants of integration defined by the boundary conditions of the beam. For a simply supported beam, these constants are set so that rotation is zero at mid-span and deflection is zero at its supports.

For the purposes of this paper, a maximum deflection constraint is placed on the optimization using Eq. (28):

$\Delta_{\max }-L / 360 \leq 0$.

\section{Multiple load cases}

When designing for multiple load cases, this paper's method can be used one of two ways. The first is to design a shape optimized beam for each load case, and then chose the most conservative design that meets all of the load cases. The second is to optimize the design for the dominant load case and then check the final design against the other load cases. In most structural applications, the dominant load case for beams is a uniform load. Consequently, the results discussed in the following section assume a dominant load case of a uniform line load.

\section{Results}

This section demonstrates the flexibility of this method to account for design choices such as varied support locations, self weight, non-rectangular section geometries, and inelastic material systems like wood and reinforced concrete. A number of trial optimizations are carried out to prove the viability and flexibility of the shape optimization method detailed in "A new approach to beam shaping" section, and the resultant designs and volume reductions are presented here.

\section{Analytical benchmarks}

First, a series of shape optimizations compare the results of this method to the results of the analytical solutions in "Analytical solution: direct solve of uniform strength beams" section. This is done by minimizing the volume of a beam, defined by the variable configuration and bounds shown in Fig. 11, and subject to the shear and moment constraints described in "Constraints" section. The results of this shape optimization are summarized in Table 2.

Volume reductions are close to those of the analytically designed beams in "Analytical solution: direct solve of uniform strength beams" section, but not exact. This difference is due to a loss in precision when interpolating between few variable sections rather than designing sections at smaller increments along the beam's length (see Fig. 12), but the results can be slightly improved by adding more variable sections. Nonetheless, meaningful volume reductions are attainable through this method.

Table 2 Comparison between the results of analytical solution and numerical optimization

\begin{tabular}{lll}
\hline Type of shaped beam & $\begin{array}{l}\text { Reduction in volume compared to } \\
\text { prismatic beam }\end{array}$ \\
\cline { 2 - 3 } & $\begin{array}{l}\text { Analytical solution } \\
(\%)\end{array}$ & $\begin{array}{l}\text { Numerical } \\
\text { optimization } \\
(\%)\end{array}$ \\
\hline Constant width & 20 & 20 \\
Constant depth & 30 & 28 \\
Constant aspect ratio & 24 & 22 \\
\hline
\end{tabular}


Fig. 12 Comparison between the analytical solution and numerical optimization of a constant width beam

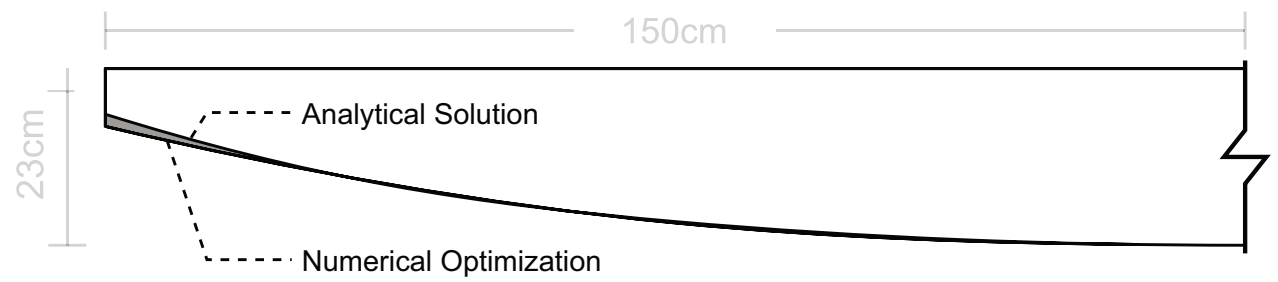

\section{Designing for varying support locations}

This method can take advantage of the parametric controls in Rhino and Grasshopper, allowing designers to iterate through numerous design choices with an understanding of their performative impacts. For example, Fig. 13 illustrates the effect of changing the support conditions by iteratively moving the first support towards the midspan by $10 \%$ of the beam's length and optimizing for the resultant load distribution. Each beam is designed for the same uniform line load, but the changing support location alters the moment and shear envelope, resulting in fairly efficient and expressive beam designs. In this example the aspect ratio was kept below six to prevent overly narrow sections that would require a check for lateral torsional stability, which is outside the scope of this paper. This results in volume reductions of $29-73 \%$ depending on the location of the support.

\section{Designing for beam self weight}

The iterative nature of numerical optimization also allows for the inclusion of a beam's self weight in its design. This is done by segmenting the beam along its length, calculating the volume and weight of each section, and applying those weights as linear loads at each segment to approximate the dead load distribution. As the beam geometry and weight distribution changes with each iteration, the load envelope can be accounted for in the next step of the optimization.
An example of this is shown in Fig. 14 below, where five segments are used to approximate the beam's self weight load distribution. The shaped optimization results in a $32 \%$ volume reduction compared to a prismatic beam designed for the same combination of live and dead load.

\section{Designing for alternative section geometries}

As described earlier, a beam's section geometry can be defined in a number of ways resulting in different parameter configurations. Figure 15 below shows a few examples designed with two variable sections along the beam's length. The difference between each beam is the shape of the variable sections. As long as care is taken to avoid self-intersecting geometry there is an infinite number of potential section configurations that may respond to fabrication constraints or material mechanics. The calculations for shear and moment capacities are adjusted to account for non-rectangular sections, but as discussed in "Shear design" section further work is required to accurately determine the maximum shear location in variable and non-rectangular section beams.

These results discuss the design of simply-supported beams made of a non-specific material with the properties described in Table 1. The following section describes how this shape optimization method is applied to common building elements such as wood beams and concrete slabs by considering their material behavior.
Fig. 13 Shape optimization results due to varying location of one support, resulting in a $29-73 \%$ reduction in beam volume depending on the location of support 1

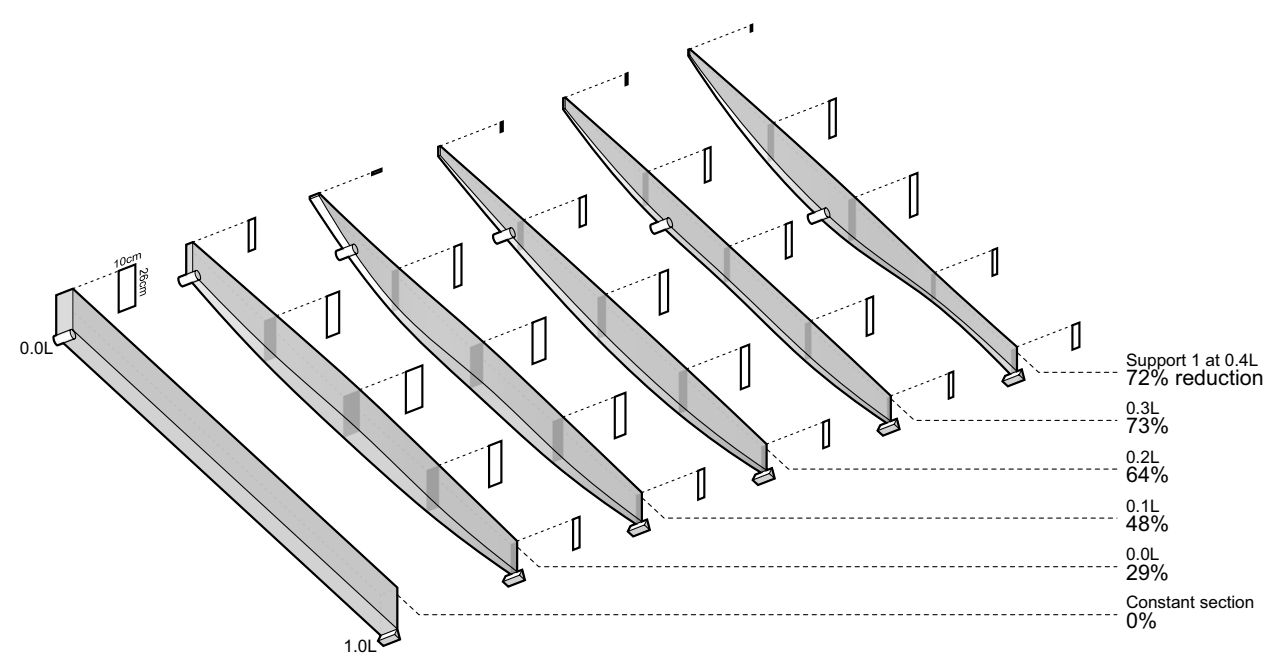


Fig. 14 Shape optimization of a beam under uniform load and approximate self weight
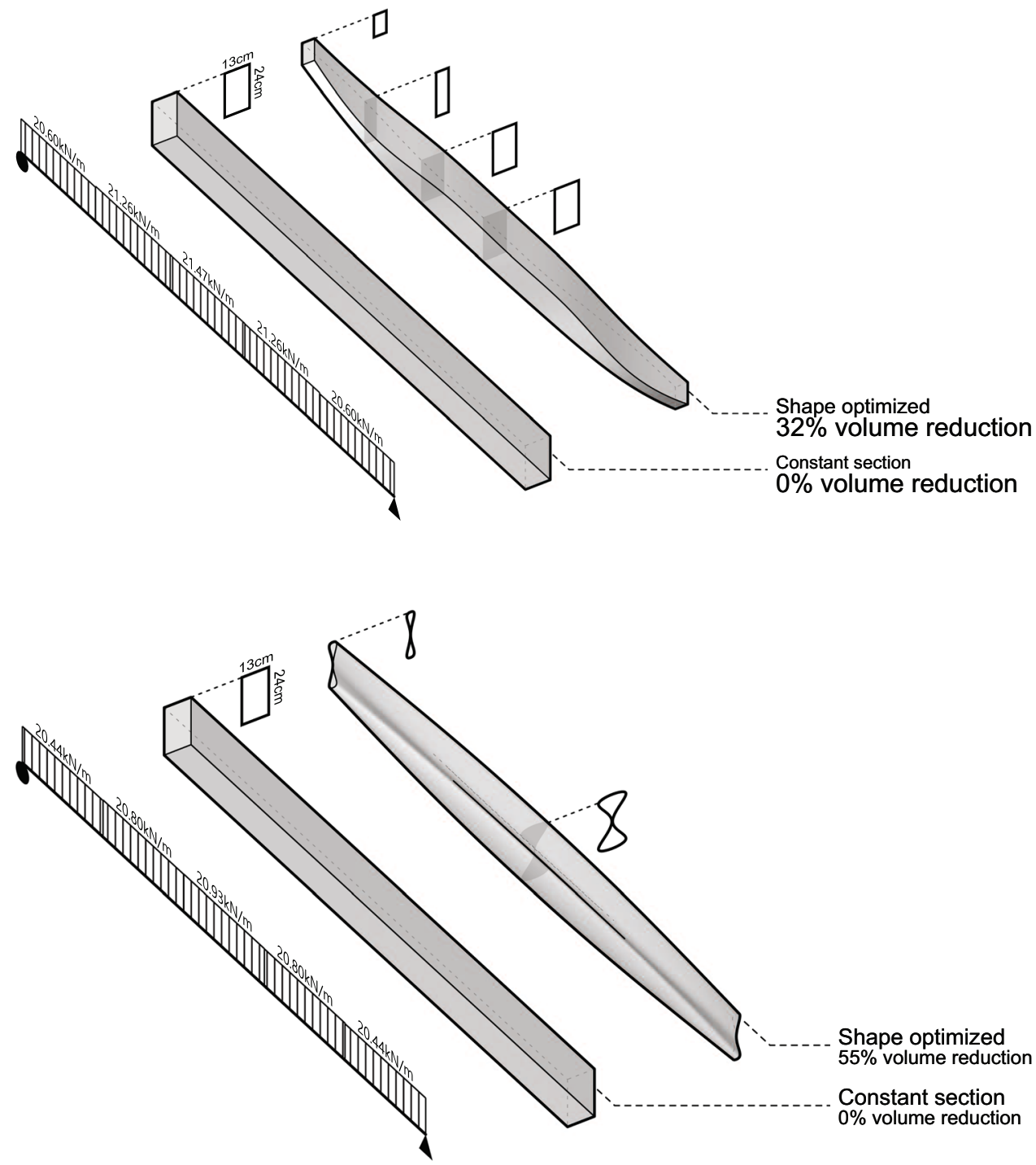

Fig. 15 Potential shape optimization results of beams with alternative cross-sections

\section{Material systems}

This paper discusses a variety of methods that use observation, analytical calculation, and numerical analysis and optimization to design materially efficient shaped beams. Nonetheless, these methods avoid most of the complexity of structural materials by assuming linear elasticity and homogenous properties throughout the beams. While these may be safe assumptions for some elements such as homogenous steel beams [18], these methods are not applicable to common material systems like wood and reinforced concrete. Consequently, this section discusses ongoing research in wood and concrete beam design that uses the method described in this paper.

In addition to including the mechanics of specific material systems in beam shape optimization, it is also important to consider new manufacturing technologies that can enable the economical materialization of structurally shaped elements. For example, additive manufacturing is being actively researched for a number of structural materials, including reinforced concrete and carbon fiber [34, 35]. Other technologies of relevance to shaped beams include robotic assembly [36], which could be used to digitally fabricate shaped glue laminated beams, and subtractive milling, which could be effective if the waste steams for the removed materials are considered.

\section{Reinforced concrete beams}

Due to its dense structural matrix, unreinforced concrete is exceptionally strong in compression; yet its brittle and porous nature makes it very ineffective against tensile stress, requiring tensile reinforcement. Existing optimization techniques typically neglect the complexity of reinforced concrete as a configuration of various 
materials - aggregate, steel, cement, water-resulting in excessive material use and uncertain results.

Research by the authors applies a method similar to the one described in this paper to reinforced concrete beam design. This method uses numerical optimization and analytical structural design to determine efficient concrete beams and slab geometries [37]. The structural analysis references standard concrete design codes [38, 39] and research in variable section concrete beam behavior [40]. Focusing on residential construction in the Global South, this research references digital design and fabrication methods that are relevant to the construction industries in developing countries. While this method may result in more complex formwork, it also allows for the constraints of available fabrication methods (like curved steel plates or injection molded PVC) to inform the optimization results. Further research is needed to quantify the cost and embodied carbon of more complex formwork, but steps can be taken to reduce those costs at scale, such as using reusable forms made from steel or plastic or restricting the curvature of steel reinforcement. To date, this work has shown that mass and embodied energy reductions of over $60 \%$ (compared to one-way flat slabs) are attainable in residential floor construction without altering the materials used in common construction (see Fig. 16).

\section{Timber beams}

There is increasing interest in the adoption of timber construction as a low-carbon substitute. The growing availability of digital fabrication and customizable mass timber products offer an opportunity for material efficiency in timber structures as well, such as shaped timber beams, which can offer cost savings in applications where material costs dominate, such as mass timber.

Structural optimization of timber elements needs to consider the anisotropic structural behavior of the material. The strength of the material is mainly defined by its mechanical properties in the axis parallel to the longitudinal grain direction and the axis perpendicular to the grain direction. Both needs to be carefully analyzed, especially in shaped elements made of timber.

Research by the authors applied the presented numerical methods to timber beams [21]. The research looked at the material saving opportunities for a wide range of beam types (width and height ratio) and beam spans. Depending on the shaping approach and the number of variables, savings up $50 \%$ of structural material (or more in extreme cases) can be realized (see Fig. 17).

Shape optimized timber elements require subtractive CNC milling or advanced additive manufacturing techniques; these opportunities, and the environmental and economic costs associated with them, are discussed in previous literature [21]. In the future, the use of shaped timber
Fig. 16 Rendering of shaped concrete slab for housing in India, designed for continuous span of $8.3 \mathrm{~m}$ and a $70 \%$ embodied energy reduction when compared to a prismatic slab

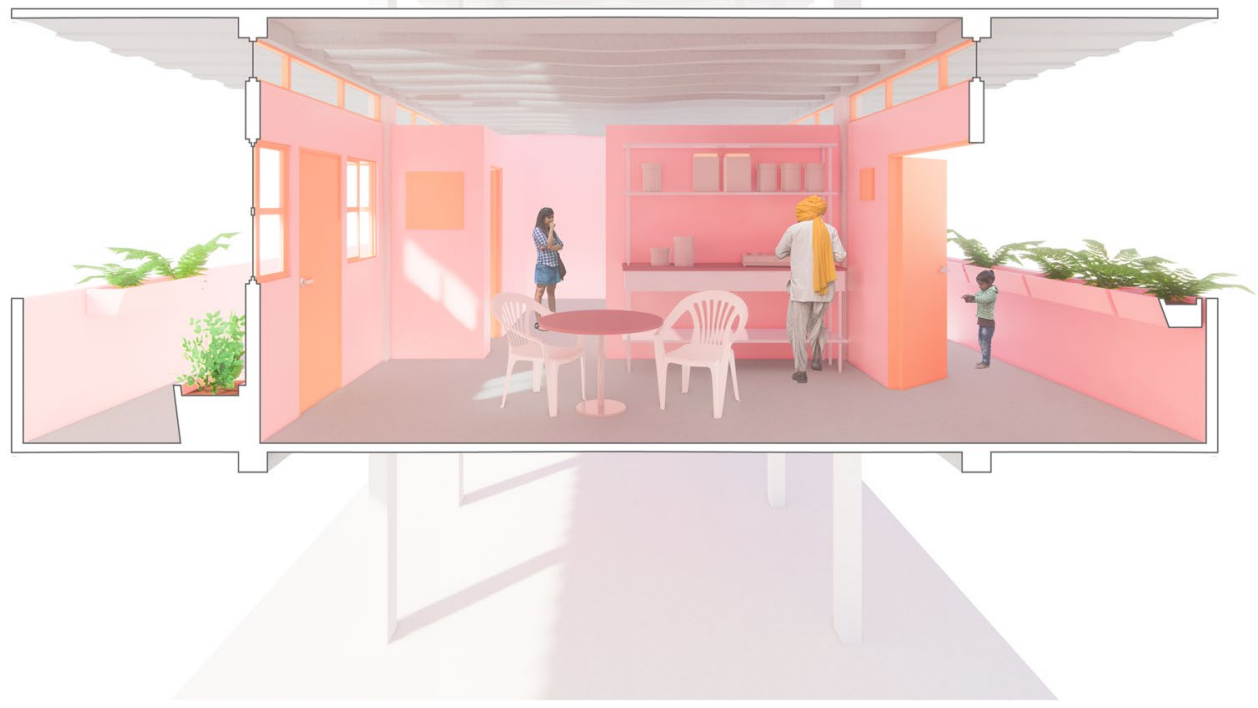


Fig. 17 Rendering of potential design of shape optimized timber beams (image by Natalie Bellefleur)

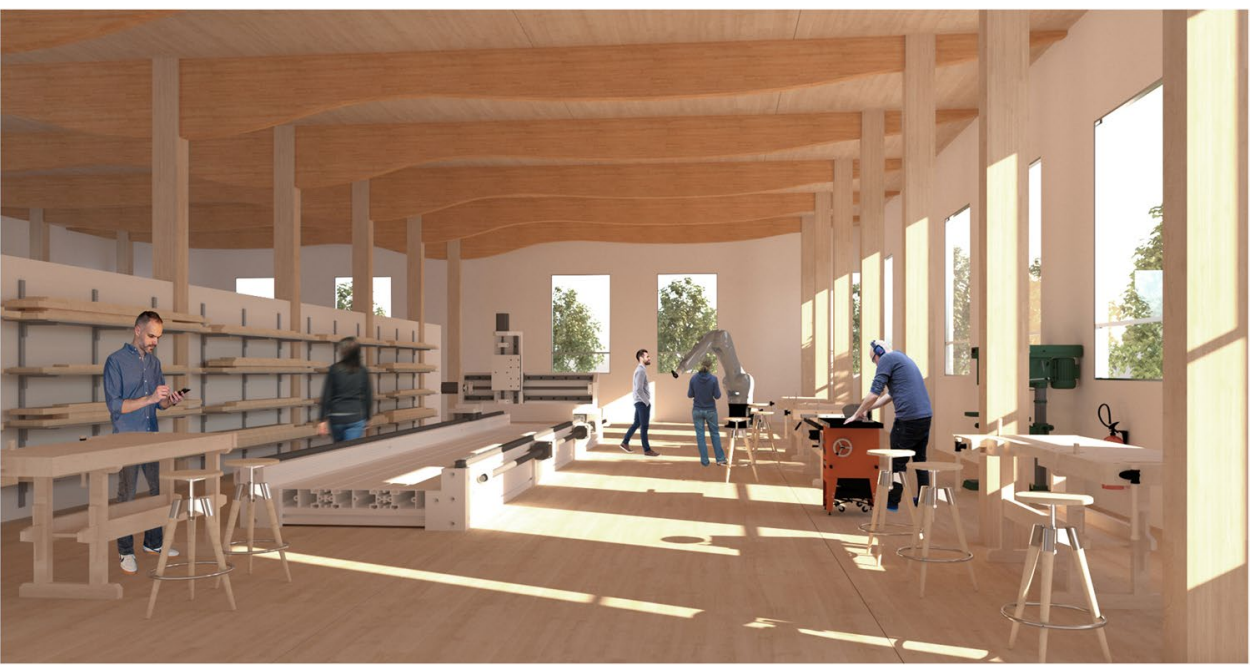

elements can be used to shape to a variety of structural requirements, such as larger section sizes around mechanical connections or holes to allow for building services for example, or as a design feature of the architecture.

\section{Conclusion}

This paper contributes new knowledge to the design of shaped beams by reviewing historic literature and presenting a novel method for beam shape optimization. After discussing the numerous approaches to shaped beam design up until the mid-twentieth century, the paper provides an overview of several methods for the design of shaped beams. This overview begins with an analytical direct-solve approach, relating the loading envelope to a single variable dimension along the length of a beam, and then summarizes more advanced methods of optimization. Finally, the paper discusses the limitations of these methods and the research being done to overcome these limits by pairing numerical optimization with analytical structural design. This allows for more geometric flexibility and the inclusion of constraints such as fabrication methods and material behavior. This method is utilized in the shape optimization of wood and concrete elements using new tools for numerical optimization in architecture design environments such as Rhino.

\section{Potential impact}

The embodied energy of construction accounts for $11 \%$ of the global carbon emissions, and that number is only expected to rise [41]. With the expected demand for construction and the accelerating climate crisis, new practices are necessary to curb the ecological costs of our buildings. Structural elements typically account for $50 \%$ of the mass and embodied energy of a building and, as a building exceeds 10 stories, the horizontal spanning elements can account for over $80 \%$ of the structural mass and embodied energy [42]. By reducing the material use of horizontal spanning elements, the weight and embodied energy could be substantially reduced. This would have the added benefits of reducing the load on vertical elements and decreasing the demand on building foundations. Additionally, lateral loads like seismic activity would be reduced proportional to a reduction in the weight of a building. Future work may also bolster the potential impact of this method by building on the work of Rozvany [43] and optimizing full-building beam and column layouts, assuming shape-optimized beam geometries.

\section{Concluding remarks}

In the past, high material costs and affordable labor demanded an architecture of material efficiency. This brought performance-driven design to the forefront and elevated structural design to a form of art. Today, the rising ecological costs of construction and newfound fabrication technologies are driving a new demand for materially efficient design. Emerging methods of fabrication enable designers to realize increasingly complex designs and processes, providing a pathway to constructing the oft-complex results of structural optimization. This becomes especially useful when the architectural design of a structure can reflect, rather than obscure, the resolution of forces. These methods may enrich the design of the built environment by pairing architectural aesthetic with structural performance. The strategies presented in this paper take advantage of these new tools and apply them to existing processes of structural analysis, resulting in a novel approach to structural design. The goal of this research is to enable a new era of high-performance design in architecture, beginning with the simple beam. 
Acknowledgements Funding for this research was provided by the Dar Group Urban Seed Grant Program at the Norman B. Leventhal Center for Advanced Urbanism, Massachusetts Institute of Technology.

\section{References}

1. Detter D (2018) How cities can lead the way in bridging the global housing gap. World Economic Forum. https://www.weforum.org/ agenda/2018/06/cities-global-housing-gap-dag-detter/. Accessed 19 Sept 2020

2. Abergel T, Dean B, Dulac J (2017) Global Status Report 2017: Towards a zero-emission, efficient, and resilient buildings and construction sector. U. N. Environ. Programme, p 48

3. De Wolf CEL (2017) Low carbon pathways for structural design: embodied life cycle impacts of building structures. Thesis, Massachusetts Institute of Technology. Accessed 27 Sept 2017. [Online]. http://dspace.mit.edu/handle/1721.1/111491

4. Foraboschi P, Mercanzin M, Trabucco D (2014) Sustainable structural design of tall buildings based on embodied energy. Energy Build 68(Part A):254-269. https://doi.org/10.1016/j.enbuild.2013.09.003

5. Anderson S (2004) Eladio Dieste: innovation in structural art, 1st edn. Princeton Architectural Press, New York

6. Raj M, Mehta V, Mehndiratta RR, Huber A (eds) (2016) The structure: works of Mahendra Raj. Park Books, Zurich

7. Bechthold M (2003) On shells and blobs—structural surfaces in the digital age. Harv Des Mag 19. [Online]. Available: https:// www.researchgate.net/publication/297452900_On_shells_and_ blobs_-_Structural_surfaces_in_the_digital_age. Accessed 14 Mar 2018

8. Timoshenko S (1953) History of strength of materials: with a brief account of the history of theory of elasticity and theory of structures. Dover Publications, New York

9. Timoshenko S (1930) Strength of materials. Krieger Pub. Co., Malabar

10. Muttoni A (2011) The art of structures: introduction to the functioning of structures in architecture. EPFL Press

11. Opatowski I (1945) Cantilever beams of uniform strength. Q Appl Math 3(1):76-81

12. Li JP, Gross WA (1955) Beams of uniform strength subjected to uniformly distributed load. Proceedings of the Iowa Academy of Science 62(1)

13. Barnett RL (1963) Minimum-weight design of beams for deflection. Trans Am Soc Civ Eng 128(1):221-255

14. Haftka RT, Gürdal Z, Kamat MP (1990) Elements of structural optimization. Springer Netherlands, Dordrecht. [Online]. Available: https://doi.org/10.1007/978-94-015-7862-2. Accessed 20 Nov 2018

15. Haug EJ Jr, Kirmser PG (1967) Minimum weight design of beams with inequality constraints on stress and deflection. J Appl Mech 34(4):999-1004. https://doi.org/10.1115/1.3607869

16. Dupuis G (1971) Optimal design of statically determinate beams subject to displacement and stress constraints. AIAA J 9(5):981-984

17. Heyman $\mathbf{J}$ (1959) On the absolute minimum weight design of framed structures. Q J Mech Appl Math 12(3):314-324. https:// doi.org/10.1093/qjmam/12.3.314

18. Barnett RL (1966) Survey of optimum structural design. Exp Mech 6(12):19A-26A

19. Maki AC, Kuenzi EW (1965) Deflection and stressses of tapered wood beams. Forest Products Laboratory

20. Paglietti A, Carta G (2009) Remarks on the current theory of shear strength of variable depth beams. Open Civ Eng J 3(1):28-33

21. Mayencourt P, Mueller C (2020) Hybrid analytical and computational optimization methodology for structural shaping: materialefficient mass timber beams. Eng Struct 215:110532
22. Haftka RT, Grandhi RV (1986) Structural shape optimization-a survey. Comput Methods Appl Mech Eng 57(1):91-106

23. Imam MH (1982) Three-dimensional shape optimization. Int J Numer Methods Eng 18(5):661-673

24. Braibant V, Fleury C (1984) Shape optimal design using B-splines. Comput Methods Appl Mech Eng 44(3):247-267

25. Veenendaal D, Coenders J, Vambersky J, West M (2011) Design and optimization of fabric-formed beams and trusses: evolutionary algorithms and form-finding. Struct Concr 12(4):241-254

26. Orr JJ, Darby A, Ibell T, Evernden M (2014) Design methods for flexibly formed concrete beams. Proc Inst Civ Eng - Struct Build 167(11):654-666

27. Rechenraum e.U.-goat (2018). https://www.rechenraum.com/en/ goat.html. Accessed 6 May 2018

28. Brown NC, Jusiega V, Mueller CT (2020) Implementing datadriven parametric building design with a flexible toolbox. Autom Constr 118:103252

29. Powell MJD (2007) A view of algorithms for optimization without derivatives. Cambridge University Technical Report DAMTP, p 12

30. Barnhill RE (1977) Representation and approximation of surfaces. In: Rice JR (ed) Mathematical software, Elsevier, pp 69-120. https://doi.org/10.1016/B978-0-12-587260-7.50008-X

31. Taylor JE (1969) Maximum strength elastic structural design. J Eng Mech Div 95(3):653-664

32. Makky SM, Ghalib MA (1979) Design for minimum deflection. Eng Optim 4(1):9-13

33. Ismail MA, Mueller CT (2019) A platform of design strategies for the optimization of concrete floor systems in India. In: Cruz PJS (ed) Structures and architecture: bridging the gap and crossing borders, 1st edn. CRC Press, pp 399-408. https://doi.org/10.1201/ 9781315229126-47

34. Buswell RA, Leal de Silva WR, Jones SZ, Dirrenberger J (2018) 3D printing using concrete extrusion: a roadmap for research. Cem Concr Res 112:37-49

35. Paolini A, Kollmannsberger S, Rank E (2019) Additive manufacturing in construction: a review on processes, applications, and digital planning methods. Addit Manuf 30:100894. https://doi.org/ 10.1016/j.addma.2019.100894

36. Huang Y, Garrett CR, Mueller CT (2018) Automated sequence and motion planning for robotic spatial extrusion of 3D trusses. Constr Robot. https://doi.org/10.1007/s41693-018-0012-z

37. Ismail MA, Mueller CT (2021) Minimizing embodied energy of reinforced concrete floor systems in developing countries through shape optimization. Eng Struct 246:112955. https://doi.org/10. 1016/j.engstruct.2021.112955

38. ACI Committee 318, ACI Committee 318, ACI Committee 318, and American Concrete Institute (2016) Building code requirements for structural concrete (ACI 318-14): an ACI standard; Commentary on building code requirements for structural concrete (ACI 318R-14): an ACI report

39. Bureau of Indian Standards (2000) IS 456: Plain and reinforced concrete-code of practice

40. Hawkins WJ et al (2016) Flexible formwork technologies-a state of the art review. Struct Concr 17(6):911-935

41. Architecture 2030 (2018) New buildings: embodied carbon. https:// architecture2030.org/new-buildings-embodied/. Accessed 30 Jun 2021

42. Block P, Paulson N (2019) E4: efficiency, economy, elegance, and ecology. In: Design - tales of science and innovation, Chronos Verlag, pp 13-21

43. Rozvany GIN (1976) Optimal design of flexural systems. Elsevier. https://doi.org/10.1016/C2013-0-02754-5

Publisher's Note Springer Nature remains neutral with regard to jurisdictional claims in published maps and institutional affiliations. 